\title{
Precision Medicine, Artificial Intelligence, and Genomic Markers in Urology. Do we need to Tailor our Clinical Practice?
}

\section{Medicina de precisión, inteligencia artificial y marcadores genómicos en urología. ¿Debemos cambiar nuestra práctica clínica?}

\author{
Julián Chavarriaga ${ }^{1}$ Camila Moreno $^{1 \odot}$
}

\begin{abstract}
Address for correspondence Julian Chavarriaga, MD, Division of Urology, Hospital Universitario San Ignacio, Pontificia Universidad Javeriana, Carrera 7, 40/62, Bogotá, Colombia (e-mail: chavarriagaj@javeriana.edu.co).
\end{abstract}

\begin{abstract}
Precision medicine plays a key role in urological oncology practice nowadays, with the breakthrough of the poly (ADP-ribose) polymerase inhibitors (PARPi), which play a critical role in different DNA damage repair (DDR) pathways, the immune checkpoint inhibitors, the genomic expression profiles and current genome manipulation-directed targeted therapy. Information and technology (IT) are set to change the way we assess and treat patients and should be reviewed and discussed. The aim of the present article is to demonstrate a detailed revision on precision medicine, including novel therapeutic targets, genomic markers, genomic stratification of urological patients, and the top-notch technological breakthroughs that could change our clinical practice

We performed a review of the literature in four different databases (PubMed, Embase, Lilacs, and Scielo) on any information concerning prostate, bladder, kidney and urothelial cancer novel treatments with PARPi, immune checkpoint inhibitors (ICls), targeted therapy with fibroblast growth factor receptor inhibitors (FGFRi), and theranostics with prostatespecific membrane antigen (PSMA) targeted monoclonal antibodies. Artificial intelligence, machine learning, and deep learning algorithm in urological practice were also part of the search. We included all articles written in English, published within the past 7 years, that discussed outstanding therapies and genomics in urological cancer and artificial intelligence applied to urology. Meanwhile, we excluded articles with lack of a clear methodology and written in any other language than English.

One-hundred and twenty-six articles of interest were found; of these, 65 articles that presented novel treatments of urological neoplasms, discussed precision medicine, genomic expression profiles and biomarkers in urology, and latest deep learning and machine learning algorithms as well as the use of artificial intelligence in urological
\end{abstract}

received

February 19, 2020

accepted

May 26, 2020
DOI https://doi.org/

$10.1055 / \mathrm{s}-0040-1714148$.

ISSN 0120-789X.

e ISSN 2027-0119.
Copyright (c) 2020, Sociedad Colombiana License terms de Urología. Publicado por Thieme Revinter Publicações Ltda., Rio de Janeiro, Brazil. Todos los derechos reservados. 
practice were selected. A critical review of the literature is presented in the present article.

Urology is a constantly changing specialty with a wide range of therapeutic breakthroughs, a huge understanding of the genomic expression profiles for each urological cancer and a tendency to use cutting-edge technology to treat our patients. All of these major developments must be analyzed objectively, taking into account costs to the health systems, risks and benefits to the patients, and the legal background that comes with them. A critical analysis of these new technologies and pharmacological breakthroughs should be made before considering changing our clinical practice. Nowadays, research needs to be strengthened to help us improve results in assessing and treating our patients.

\section{Resumen}

\section{Keywords}

- medicina de precision

- genetica

- genomica

- inteligencia artificial

- teranosticos

- cancer
La medicina de precisión juega un rol fundamental en la práctica clínica de la urologia oncológica en la actualidad, con el desarrollo de los inhibidores de la poli (ADP-ribosa) polimerasa (PARPi), que juegan un papel fundamental en las distintas vías del reparo del ADN dañado (RAD), los inhibidores del punto de chequeo inmune (ICI), los perfiles de expresión genómicos, y la terapia blanco-dirigida a la manipulación genómica. El desarrollo tecnológico y la informática están cambiando la forma como evaluamos y tratamos a los pacientes, y se debe discutir y revisar a detalle. El objetivo de este artículo es hacer una revisión detallada acerca de la medicina de precisión, genómica, y los avances tecnológicos en nuestro campo.

Realizamos una revisión de la literatura en cuatro bases de datos diferentes (PubMed, Embase, Lilacs, y Scielo), buscando cualquier información relacionada con cáncer de próstata, vejiga, riñón y carcinoma urotelial, tratamientos novedosos con PARPi, ICl, terapia-blanco con inhibidores del receptor del factor de crecimiento de los fibroblastos (FGFRi) y teragnósticos con anticuerpos monoclonales dirigidos al antígeno de membrana específico de la próstata (AMEP). Inteligencia artificial, aprendizaje de máquinas y algoritmos de aprendizaje profundo en la práctica urológica también fueron revisados. Incluimos artículos escritos en inglés, publicados dentro de los últimos 7 años, que abordaran terapias novedosas y genómica en cáncer urológico e inteligencia artificial aplicada a la urología. Excluimos artículos con falta de una metodología adecuada y escritos en cualquier idioma diferente al inglés.

En total, 126 artículos de interés fueron encontrados, y, de estos seleccionamos 65 artículos que reportaban tratamientos novedosos para neoplasias urológicas, discutían medicina de precisión y perfiles de expresión genómica y bio-marcadores en urología, algoritmos de aprendizaje profundo, aprendizaje de máquina, y el uso de inteligencia artificial en la práctica urológica. Se hizo una revisión crítica de la literatura que se presenta en este artículo.

La urología es una especialidad constantemente en cambio, con un gran rango de avances terapéuticos, un gran conocimiento de los perfiles de expresión genómica para cada cáncer urológico, y una tendencia a utilizar tecnología de punta para estudiar y tratar a nuestros pacientes. Todos estos desarrollos se deben analizar objetivamente, y hay que tener en cuenta los costos al sistema de salud, los riesgos y beneficios para los pacientes, y el contexto legal que implica cada uno. Hasta la fecha, estos avances tecnológicos y farmacológicos se deben analizar con cautela antes de vernos en la posición de cambiar nuestra práctica clínica. Se debe fortalecer la investigación médica para mejorar los resultados en el tratamiento y abordaje de nuestros pacientes. 


\section{Introduction}

New frontiers in urology are set further beyond with the new technological, genomics and pharmaceutical developments in this field. Urological practice has changed considerably in the last decade, new therapeutic targets have been studied, including manipulation of the immune system to attack cancer cells. ${ }^{1,2}$ The genomic expression profiles and genome manipulation-directed targeted therapy are constantly changing our practice and are set to be the base of a tailored approach for each patient's genomic aberrations, also known as precision medicine. ${ }^{1,3-6}$

Technology has had a major influence in urology in recent years, initially with the adoption of electronic records of the clinical history and with picture archiving and communication systems (PACS). ${ }^{5,7-9}$ Followed with the development of deep learning algorithms, which are multilayered neural networks that learn from vast amounts of data, machine learning, which are algorithms fed by the exposition of data over time, with constantly improving performance and artificial intelligence (AI) which are computerized programs that can sense, reason, act and adapt according to an specific situation. ${ }^{8}$ All of these cutting-edge technological tools have been studied for the treatment of urolithiasis, urological cancer, hypospadias and have been able to successfully identify renal cell carcinoma, prostate carcinoma in surgical pathology and to discriminate tumors in white light cystoscopy. $7,10-16$

The aim of the present article is to present a detailed revision on precision medicine, including novel therapeutic targets, genomic markers and genomic stratification of urological patients, and the top-notch technological breakthroughs that could change our clinical practice.

\section{Methods}

We performed a review of the literature in four different databases (PubMed, Embase, Lilacs, and Scielo) on any information concerning prostate, bladder, kidney and urothelial cancer novel treatments with poly (ADP-ribose) polymerase inhibitors (PARPi), immune checkpoint inhibitors (ICIs), targeted therapy with fibroblast growth factor receptor inhibitors (FGFRi), and theranostics with prostate specific membrane antigen (PSMA) targeted monoclonal antibodies. A search for prostatic adenocarcinoma (PCa) and urothelial carcinoma (UC) genomics was conducted. Artificial intelligence, machine learning, and deep learning algorithms in urological practice were also part of the search.

The search criteria were established in the form of free text and indexed terms. We used the MeSH terms: kidney cancer, prostate cancer, transitional cell carcinoma, poly ADPribose polymerase, antineoplastic agents, immunological, 177Lu-EB-PSMA-617, 68Ga-PSMA, intelligence, artificial, learning, deep, learning, machine, urologic diseases, urologic surgical procedures, urinary lithiasis, pathology surgical, genomics, comparative, genetic screening, and growth factors, fibroblast. The search was limited to publications in the past 7 years, and articles written in any other language but English were discarded.
A gray literature search was also performed on the pages of the National Technical Information Service (NTIS) and the European Association for Gray Literature Exploitation (EAGLE); however, no additional relevant information was found.

The articles were all original studies. References were reviewed by title and abstract by two independent reviewers. From the initial selection of articles, the references were reviewed integrally, ensuring they provided the aforementioned information of interest on all the topics. Duplicate studies were removed, and studies written in any language different from English were discarded.

\section{Discussion}

\section{Precision Medicine and Genomic Markers}

Precision medicine, despite being relatively new, is a concept that has been part of healthcare for decades. ${ }^{1,17}$ It takes into account the individual bases of genomics, lifestyle, and environment to precisely tailor personal therapeutic targets to treat disease and stratify patients to guide the best therapeutic approach. ${ }^{1,4,9,17}$

Genome instability has been described as one of the hallmarks of urological cancer. In recent years, different DNA damage repair (DDR) pathways have been found to be altered in urothelial carcinoma (UC), renal cell carcinoma (RCC), and metastatic castration-resistant prostate cancer (mCRPC). ${ }^{1-4,9,17,18}$ New targets in urological oncology are emerging at a fast pace in the era of precision medicine. ${ }^{2,17}$

Precision medicine is powered by patient data, health records, and genetic codes. Initiatives like The Cancer Genome Atlas research network (TCGA), which is a joint effort between the National Cancer Institute ( $\mathrm{NCI}$ ) and the National Human Genome Research Institute (NHGRI), have molecularly characterized over 20,000 primary cancers and generated over 2.5 petabytes of genomic, transcriptomic, proteomic, and epigenomic data, which has depicted the genomic landscape of nonmetastatic and metastatic PCa. ${ }^{3,4,17}$

Understanding of the genomic landscape in urological neoplasms has identified various dysregulated biological pathways that are relevant when determining the prognosis and natural course of the disease but that are very important for designing and using precise therapeutic targets to offer the patients a personalized management strategy according to their personal genomic alterations. ${ }^{1,2,4,17}$ Four main pharmaceutical breakthroughs in urological cancer that are changing urological clinical practice worldwide are PARPi, ICIs, FGFRi, and theranostics with PSMA-targeted monoclonal antibodies with $\beta$ and $\alpha$-emitting radioisotopes. ${ }^{1,2,4,19-21}$

Immune checkpoint inhibitors are designed to enhance or reactivate antitumor immunity. It is one of the hot topics in urological cancer nowadays, given that they could be used for treating PCa, urothelial carcinoma, and renal-cell carcinoma (RCC), amongst others. Made easy ICIs could be divided in programmed death receptor ligand 1 (PD-L1) inhibitors, cytotoxic T-lymphocyte antigen 4 (CTLA-4) inhibitors and therapeutic vaccines such as sipuleucel-T, which are autologous mononuclear cells ex-vivo activated with a recombinant fusion protein fused to granulocyte-macrophage colony-stimulating 
factor, an activator of immune cells and PROSTVAC-VF ([Sanofi, Bridgewater, $\mathrm{NJ}$ ), a cancer vaccine made of engineered poxviral vaccine targeting prostate-specific antigen (PSA)-expressing cells, both in the mCPRC scenario. ${ }^{2,4,17}$ Ipilimumab is a monoclonal antibody that targets CTLA-4, a protein receptor for downregulation of the immune system. It has been studied for the treatment of metastatic renal cell carcinoma (mRCC) in conjunction with nivolumab (Checkmate 214), and it is currently under research in mCRPC. ${ }^{2,4,17,22}$ (-Fig. 1.)

Programmed death receptor ligand 1 (PD-L1) inhibitors are human monoclonal antibodies against programmed death receptor (PD-1) blocking PD-L1 from binding to PD-1 on activated T-cells, empowering the immune system to attack neoplastic cells. ${ }^{2,4,23}$ Some of the PD-L1 inhibitors are nivolumab, atezolizumab, avelumab, and pembrolizumab. Nivolumab has been studied in mRCC, showing improved overall survival (OS) compared with tyrosine kinase inhibitors (TKIs) (CheckMate 214 , CheckMate 0.25$).{ }^{22,24}$ It has shown a meaningful clinical benefit in metastatic urothelial carcinoma, still phase 3 trials pending (CheckMate 275, CheckMate 032 ) 25,26 but has shown no benefit yet in metastatic PCa. ${ }^{2,4}$ Avelumab has been recently reported to improve progression-free survival (PFS) in combination with axitinib versus TKI alone in mRCC (JAVELIN Renal 101). ${ }^{27}$ Atezolizumab has been widely studied in metastatic urothelial carcinoma (IMvigor 211) and mRCC (IMmotion 151) with not very promising results. ${ }^{28,29}$

Pembrolizumab is the only ICI approved by the Food and Drug Administration (FDA) for solid tumors, based on the presence of defects in DNA mismatch repair (MMR) genes leading to microsatellite instability (MSI), which is associated with a high mutational burden. ${ }^{2,4,23}$ It has been reported that $12 \%$ of patients with advanced PCa have a hypermutated subtype, and it is correlated with MMR mutations (MSH2 or
MSH6), which could also be found in up to $8 \%$ of Gleason pattern 5 PCa. ${ }^{1,2,4,17}$ Pembrolizumab is currently recommended for mCRPC with MSI. ${ }^{2,4,23}$ In mRCC, it has been reported recently that pembrolizumab improves OS, and PFS in combination with axitinib (KEYNOTE-426). ${ }^{30}$ It has been extensively studied in metastatic UC as first-line therapy in cisplatin-ineligible patients (KEYNOTE-052) ${ }^{31}$ and second-line therapy after cisplatin-based chemotherapy (KEYNOTE-045), being associated with longer OS. ${ }^{32}$ Durvalumab, nivolumab, and avelumab had also been approved as monotherapy for advanced or metastatic UC after platinum-containing regimen failure. $^{18}$

Defects in DNA repair promote carcinogenesis through continued DNA replication without error correction and have been identified in up to $25 \%$ of patients with MCRPC. ${ }^{1,2,33}$ The BRCA1 and $B R C A 2$ genes are associated with higher Gleason scores, usually $\geq 8$ and higher incidence of metastatic disease. ${ }^{1,2,17}$ Germline mutations in DDR genes, in metastatic prostate cancer, differ significantly from men with localized disease (11.8 vs 4.6\%). Specific mutations are BRCA2 (5.3\%), CHEK2 (1.9\%), ATM (1.6\%), BRCA1 (0.9\%), and PALB2 (0.4\%). ${ }^{1,17}$ (-Fig. 1.)

Poly (ADP-ribose) polymerase (PARP) play a significant role in DNA repair, they work by locating the DNA defect and binding to the replication fork until the repair begins. $2,4,17,34,35$ Poly (ADP-ribose) polymerase inhibition is one of the novel pharmaceutical targets in MCRPC and many other neoplasms given that through its inhibition the single-stranded DNA breaks will become double-stranded breaks that cannot be repaired and lead to apoptosis of the tumor cells. ${ }^{2,4,17,34-36}$ The potency of trapping PARP enzymes differ significantly between inhibitors, with a trapping efficiency following a downstream fashion: talazoparib, niraparib, olaparib, rucaparib, and veliparib. $^{37}$ (-Figs. 2 and $\mathbf{3}$ )

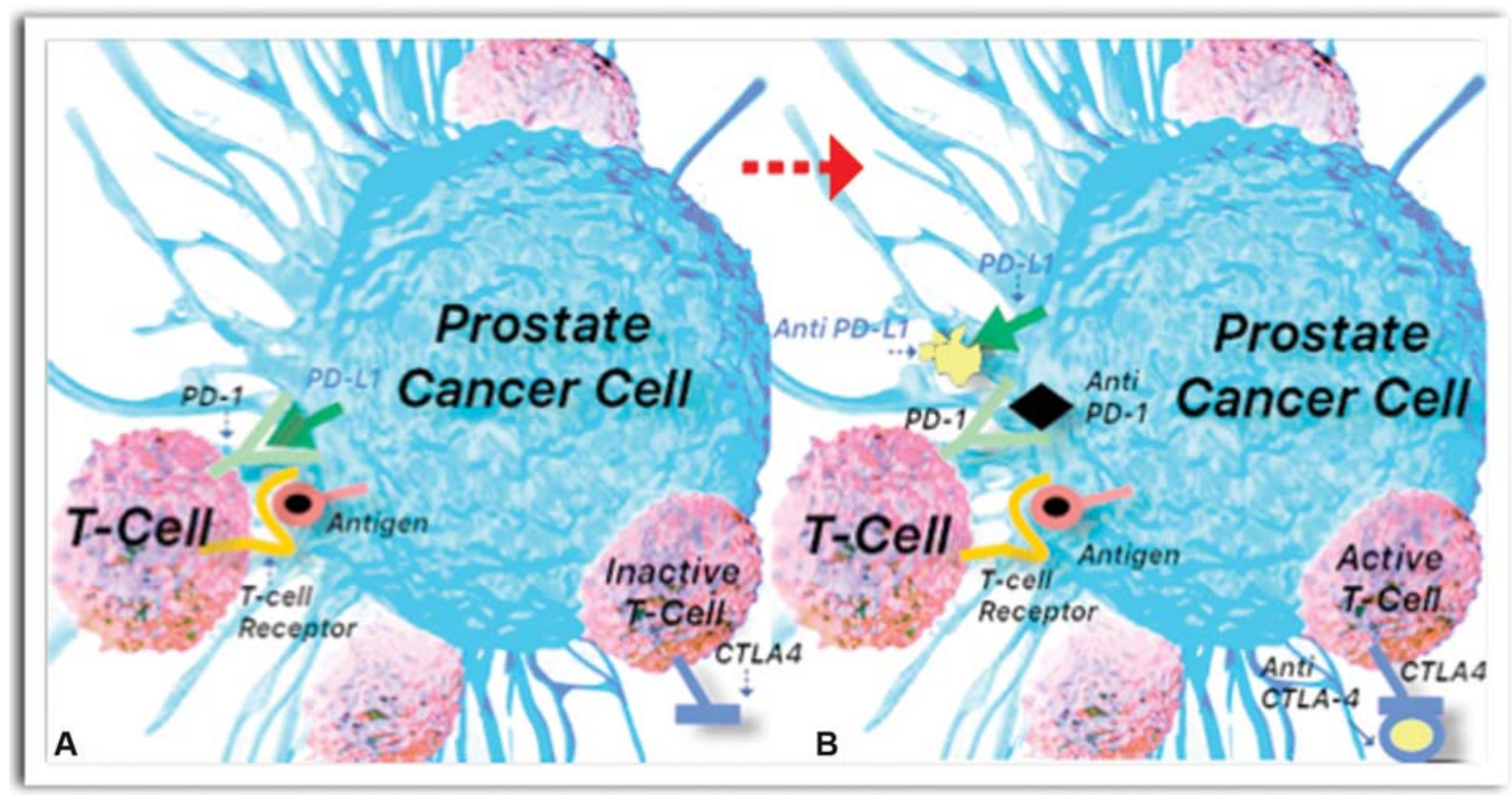

Fig. 1 (A) Prostate cancer cell expressing programmed death receptor ligand 1 (PD-L1) inhibiting T-cell from attacking; cytotoxic T-lymphocyte antigen 4 (CTLA-4), a protein receptor for downregulation blocking T-cell through antigen-presenting cell interaction. (B) Anti-programmed death receptor and anti PD-L, blocking PD-L1 from binding to PD-1 on activated T cells, empowering the immune system to attack neoplastic cells; anti-CTLA-4, activating the T-cell to attack prostatic adenocarcinoma cells. 
The last study published was the PROfound phase 3 openlabel trial, which evaluated PARPi (olaparib) in men with MCRPC who had progressed receiving an androgen-signaling-targeted inhibitor (enzalutamide or abiraterone). All patients had at least one qualifying alteration with direct or indirect role with homologous recombination repair (HRR). One arm included gene alterations in BRCA1/2 or ATM. Radiological PFS was considerably longer in the PARPi group (7.4 vs 3.6 months) (hazard ratio [HR] 0.34; 95\% confidence interval [CI], 0.25 to $0.47 ; p<0.001$ ), and $81 \%$ of control patients who had progressed crossed over to the PARPi arm. ${ }^{35,38}$ The TRITON2 study of rucaparib in patients with DDR-deficient mCRPC showed a PSA and overall response rate of $53.6 \%$ and $47.5 \%$ respectively in BRCA patients. ${ }^{36,39}$ Thus, these findings encouraged the FDA to grant priority review of these two drugs. ${ }^{36,37,39}$ The recently published TOPARP-B phase 2 trial (2019) aimed to evaluate the association between DDR gene alterations in $\mathrm{mCRPC}$ and response to olaparib $300 \mathrm{mg}$ or $400 \mathrm{mg}$. Results showed that olaparib has antitumor activity against mCRPC with DDR alterations; the composite response was $54.3 \%$ in the $400 \mathrm{mg}$ arm versus $39.1 \%$ in the $300 \mathrm{mg}$ arm, suggesting that the ideal dose should be $400 \mathrm{mg}$, twice a day (at a expense of higher toxicity). It should not go without notice that all of these patients had already received docetaxel, 88 to $92 \%$, a novel

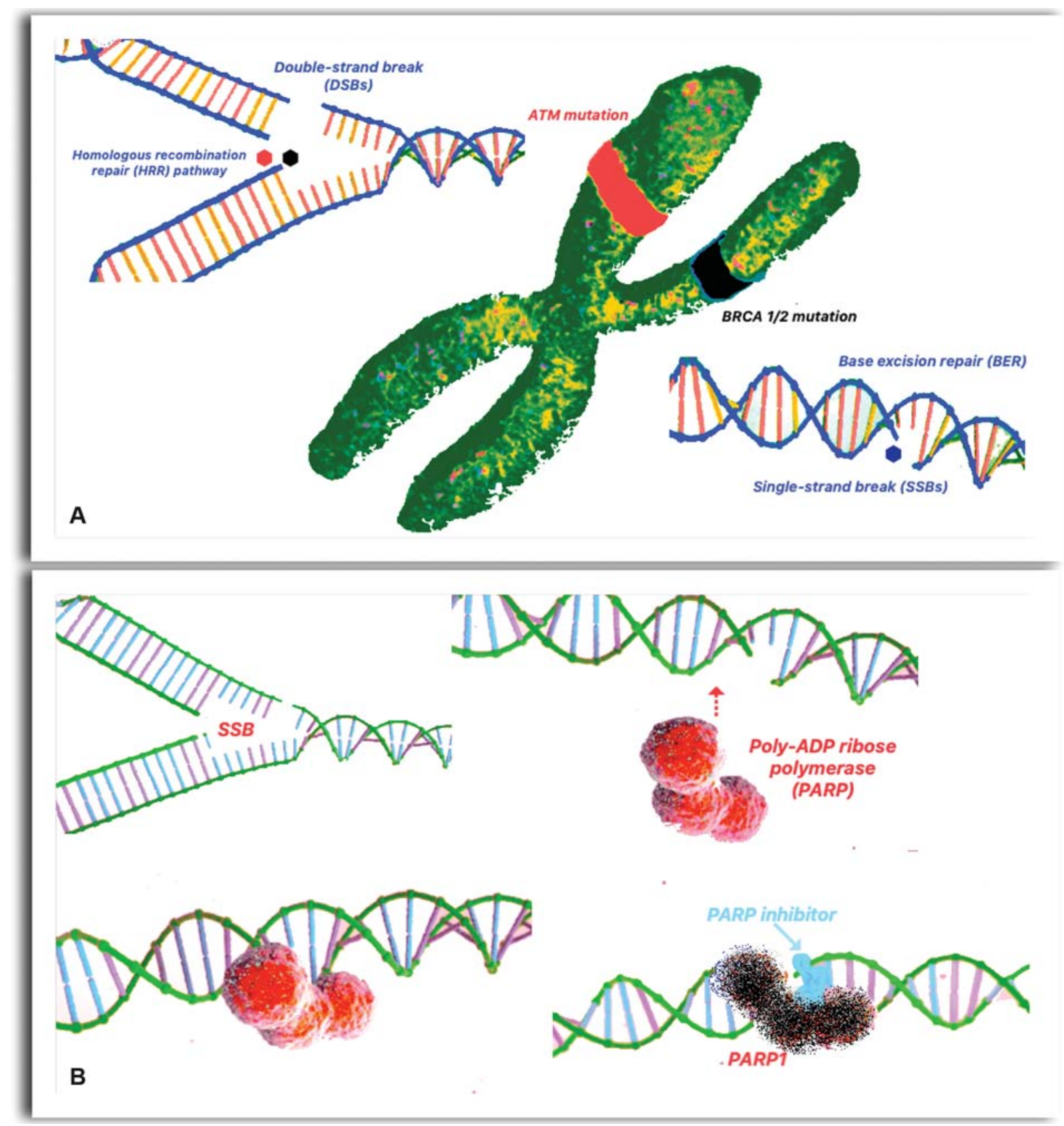

Fig. 2 (A) In human cells there are different DNA damage mechanisms. Single-strand breaks, these are repaired by the base excision repair pathway and double-strand breaks (DSBs), repaired by the homologous recombination repair (HRR) and the non-homologous end-joining pathways. BRCA $1 / 2$ and ataxia telangiectasia mutated mutations directly affect the HRR pathway. (B) Poly-ADP-ribose polymerase (PARP) is a protein that carries surveillance within the cell and recognizes DNA damage, when identified, recruits HRR to repair DNA damage. Poly-ADPribose polymerase inhibitors bind to PARP1 and prevent identification of DNA damage, leading to DNA damage accumulation and cell death. 


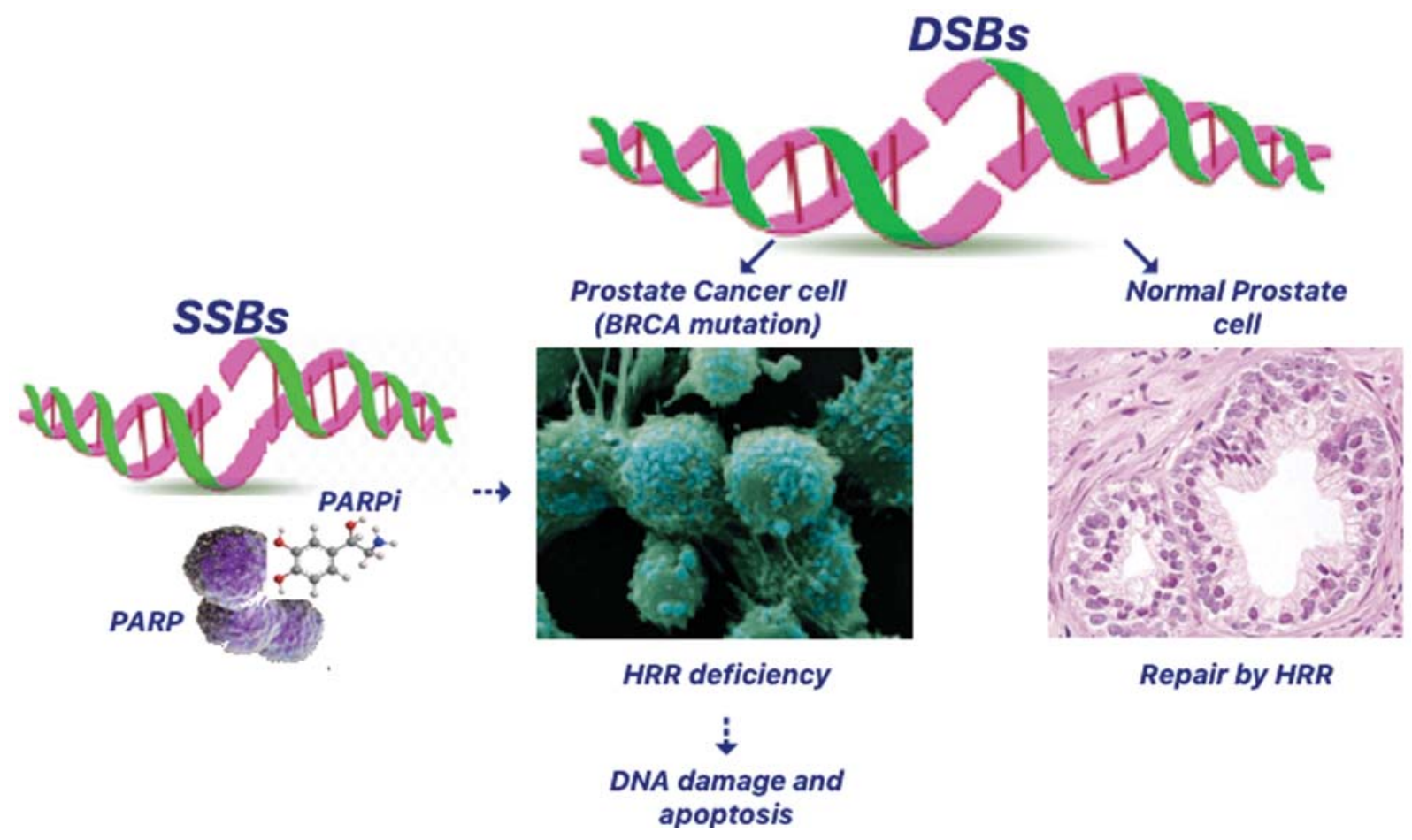

Fig. 3 Poly-ADP ribose polymerase repair DNA single-strand breaks through the base excision repair pathway. Poly-ADP ribose polymerase inhibitors, prevent repair by trapping the inactivated PARP onto the SSB, resulting in the generation of DNA double-strand breaks during the replication process. In prostate tumors with homologous recombination repair deficiency, the low-fidelity repair mechanism of non-homologous end-joining leads to DNA damage accumulation, increasing genetic instability and finally cell death.

androgen-signaling-targeted inhibitor , and 31 to $45 \%$ cabazitaxel prior to the PARPi. ${ }^{34}$

There is a rationale that PARPi in combination with androgen-signaling-targeted inhibitor could improve PSA or radiologic response rate vs androgen-signaling-targeted inhibitor alone based on the premise that the androgen receptor (AR) pathways drive progression to $\mathrm{MCRPC}$, which is due mainly to adaptive mechanisms that enable persistent AR signaling. ${ }^{37}$ Data shows that AR regulates transcription of several sets of MMR genes, PARP-1 can act as an AR co-factor and are upregulated in exposure to androgen deprivation therapy (ADT). 1,17,37

Stratification and molecular subtype identification in UC have gained popularity in recent years. The TCGA and several studies have shown that gene-expression datasets of UC suggest it could be classified in 6 molecular subtypes: NEURAL, LUMINAL, papillary-like (PAP), HER2L, mesenchymal-like (MES), squamous-cell carcinoma (SCC) and bladder carcinoma subtype of large meta-cohort databases (BOLD). ${ }^{18,40}$ Regarding muscle-invasive bladder cancer (MIBC), Choi et al identified two intrinsic subtypes: one basal and two luminal. The luminal I subtype has been associated with poor response to $\mathrm{ICI}$, given its low gene-expression associated with immune response and a poor expression of PD-L1. ${ }^{41}$ Fibroblast growth factor receptor is involved in network signaling that regulate the cell cycle through proliferation, migration and differentiation processes. ${ }^{18-20,40,41}$ Luminal MIBCs are enriched with high levels of FGFR3 and activating FGFR3 mutations. ${ }^{40,41}$ Erdafitinib, a potent TKI of FGFR1-4, was evaluated in a phase 2 clinical trial in patients with locally advanced or metastatic UC with prespecified FGFR3 or FGFR 2/3 fusion mutation, who had received at least one cycle of systemic chemotherapy or were ineligible to cisplatin. They reported a response rate of $40 \%, 37 \%$ with a partial response and with a median OS of 13.8 months and median PFS of 5.5 months, which is higher than with taxanes or vinfluvine and ICIs. ${ }^{20}$

Theranostics in PCa is another field of interest in current urological practice and are poised to transform the treatment of patients with mCRPC. ${ }^{21}$ In simple words, theranostics is the combination of a targeted therapeutic agent with diagnostic tests such as ${ }^{68} \mathrm{Ga}$-PSMA-11 PET/CT. ${ }^{21}$ VISION trial is a phase 3 multicenter prospective trial enrolling patients with progressive MCRPC with a positive ${ }^{68} \mathrm{Ga}$-PSMA-11 PET/CT, treated with at least one novel ASI and one prior taxane-based regimen. The patients are randomized to ${ }^{177} \mathrm{Lu}$-PSMA-617, a $\beta$-emitting radioisotope that releases $\beta$-particles that travel less than $2 \mathrm{~mm}$ and can irradiate small tumors in metastatic sites; at the same time, it emits low-energy gamma particles that are of utility while doing imaging-based tumor localization. ${ }^{42}$ Investigators planned to complete data collection in May 2020. ${ }^{42}$ Alpha particles targeting PSMA are in development and are a promising option for theranostics in the future, and currently a phase 1 multicenter study of the $\alpha$ particle emitter thorium-227 conjugated to a PSMA targeted monoclonal antibody is enrolling patients. ${ }^{21}$ (-Fig. 4)

\section{Artificial Intelligence, Machine learning and Deep learning algorithms}

Improvements in prediction tools for disease behavior and treatment response are a recent area of interest in urology. 

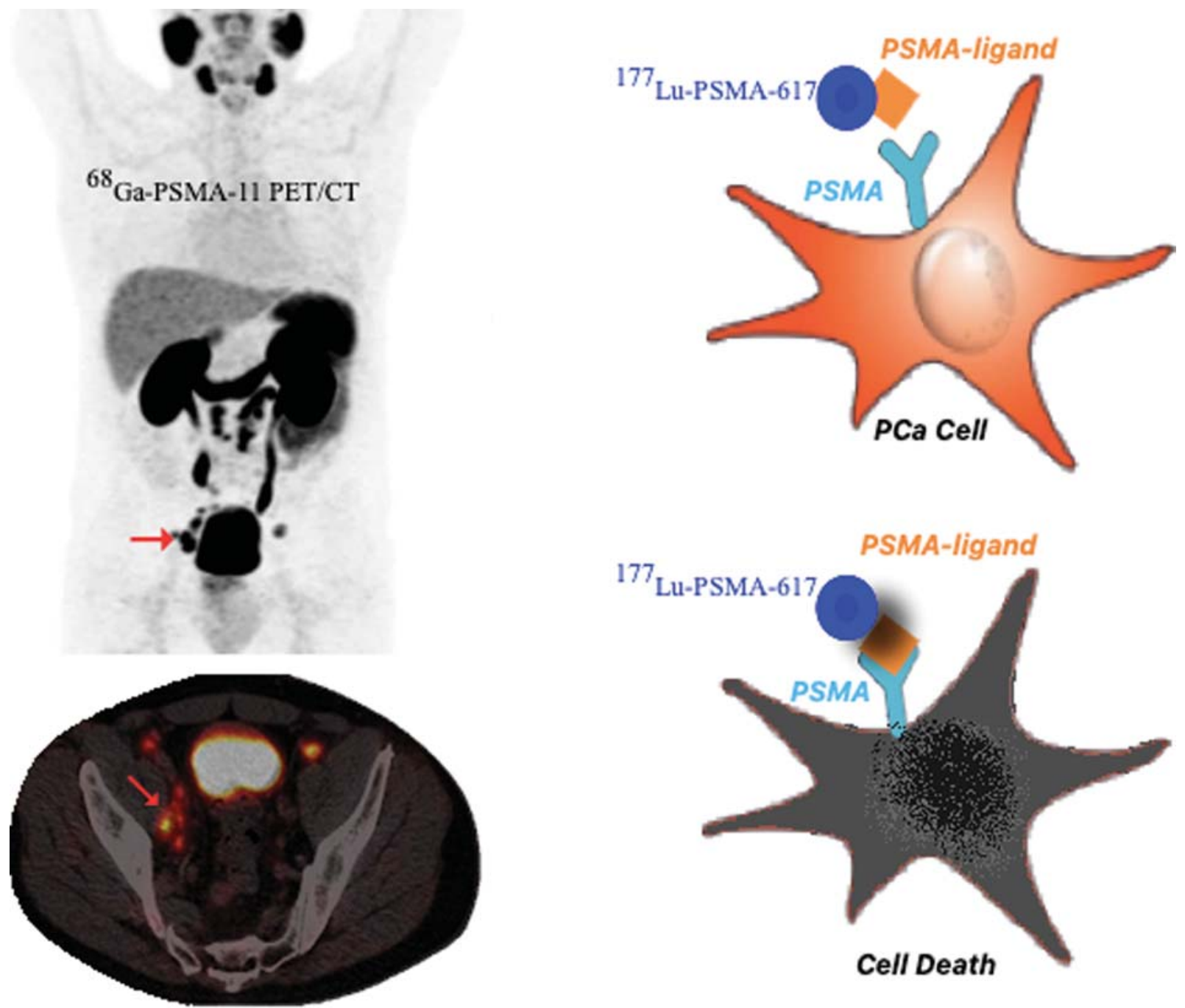

Fig. $4{ }^{68}$ Ga-PSMA-11 PET/CT with pelvic lymph nodes overexpressing prostate-specific membrane antigen (arrow). ${ }^{177}$ Lu-PSMA-617 radioisotope coupling with PSMA expressed in cancer prostatic adenocarcinoma cell, emitting $\alpha$ and gamma particles leading to tumor cell death.

Artificial intelligence systems are setting new horizons in urological practice.

A machine learns when it changes its structure in response to external information based on algorithms, which ultimately means it will improve future performance and can be applied to any situation in which repetitive data can be obtained. Artificial intelligence is the application of machine learning and uses complex mathematical models to generate conclusions. Deep learning is a subset of machine learning, based on the human neuronal structure, that can generate data driven models of biological systems. ${ }^{8}$

There are several AI methods that provide decision support systems (DSS), including Bayesian networks, expert systems, artificial neural networks (ANN), modeling systems, and decision trees.

Machine learning includes models that require the input of frequency data and previous knowledge or expert opinions, such as the Bayesian networks that allow to combine this information to arrive to an intelligent solution and expert systems that analyze information according to a series of rules or questions provided by an expert and generate conclusions. However, it cannot accommodate new questions or data.,

Deep learning uses a multi-layered structure of algorithms that correspond to artificial neurons that run in parallel and can reorganize complex patterns according to a weight that is assigned to each input and obtain "knowledge" by feeding the information repeatedly to the system (ANNs and NFMs $)^{5,8}$

The mechanisms of AI are being widely studied and developed for improving diagnostic accuracy and prediction of disease behavior in urological conditions.

Regarding prostate multiparametric magnetic resonance imaging (mpMRI), AI algorithms are focused on the automated detection of suspicious regions and are expected to reduce reader interpretation times, increase performance of nonexpert radiologists, and, ultimately, increase sensitivity and reduce inter-reader variability. ${ }^{7}$

One study found the sensitivities of mpMRI-alone and computer aided diagnosis (CAD), based on a traditional machine-learning algorithm, were similar (79\% vs $76 \%$ ), but the greatest benefit of CAD was found to be in transitional zone 
(TZ) lesions and for moderately experienced readers (84\% vs mpMRI-alone $67 \%, p=0.055)$. Times of reading improved in CAD (4.6 vs 3.4 minutes, $p<0.001$ ), and for PI-RADS.V2 of 3 or more, sensitivity was superior in the CAD group than in mpMRI-alone (72\% vs $45 \%, p<0.001) .{ }^{43}$

Deep learning algorithms have been less studied for mpMRI; however, some have proposed segmentation of the sequences to improve detection rates, assigning two parallel networks for T2 and ADC specifically, which has demonstrated an improvement in sensitivities compared with previous studies proposed. ${ }^{44}$ These studies are very promising; however, verifying the results of mpMRI with the actual pathology is the real challenge. Studies have shown that mpMRI signal characteristics are associated with tissue composition and density, specially the glandular components, which allows the creation of "radiopathomic" maps to distinguish cancerous regions. $7,45,46$

Segmentation of the histopathological components (stroma, nuclei, epithelium, lumen, etc.), which is known as semantic segmentation, is the basis of machine learning for pathology specimens. This allows to generate dichotomic results (benign vs malign) ${ }^{47}$ and further classify the tissue according to its characteristics generating a Gleason score. ${ }^{48}$ Based on these mechanisms described, deep learning systems (DLSs) have been developed to improve Gleason scoring, as follows. A retrospective study evaluated a DLS to grade prostate biopsies following the Gleason grading standard. It was designed to delineate individual glands and assign a Gleason pattern, grade, and group. This system was validated with 550 biopsies and compared with the results of 13 pathologists and 2 pathologists in training. It was tested in 579 biopsies and achieved a high correlation with the reference standard (Cohen kappa 0.918), differentiation between malignant or benign, Gleason grade, group 2 or 3, and it even outperformed 10 of 15 pathologist observers. ${ }^{10}$

Another DLS was developed using 112 million pathologist annotated image patches and validated on 331 slides, compared with pathologist experts. It demonstrated a significantly higher accuracy of $0.7(p=0.002)$ and trended to a better risk patient stratification. ${ }^{12}$

In recent years, DLSs, specially CNNs, have improved accuracy in image recognition, object detection, and semantic segmentation in kidney cancer diagnosis and classification. One study evaluated a CNN for RCC classification and survival prediction based on The Cancer Genome Atlas (TCGA) project that has resulted in digital haematoxylin and eosin (H\&E) whole-slide images (WSI) of RCC. The CNN managed to classify each RCC from normal to three subtypes of RCC (AUC 0.93), which ultimately translates in survival prediction outcomes ${ }^{14}$

Furthermore, a paper proposed a DLS for the classification of kidney cancer into subtypes using the genome of 25 types of miRNA identified to determine tumor characteristics, information provided by the TCGA. A recurrent neural network is used to classify a miRNA sample into five cancer subtypes, with an accuracy around 95\% and correlation coefficient of $0.92 .^{13}$

Regarding bladder cancer, the identification of the tumor in cystoscopy is the key for optimal transurethral resection of bladder tumor (TURBT). A study aimed to improve tumor localization and surgical resection in cystoscopy studies. With this purpose, videos with histologically confirmed tumors were selected and manually inserted into a platform based on convolutional neural networks constructed (CystoNet). Ninety-five patients were used for algorithm training, 5 for testing, and then it was validated in 54 patients. The sensitivity and specificity per-frame of CystoNet was $90.9 \%$ and 98.6, respectively (detected 39 of 41 papillary and 3 of 3 flat bladder cancers). ${ }^{11}$

Another DLS aims to predict the survival according to bladder cancer subtypes, using TCGA dataset of mRNA, miRNA, and methylation to infer two survival subtypes and apply it to any new individual sample. The high-risk survival subgroup had KRT6/14 overexpression and PI3K-Akt pathways. ${ }^{15}$

Urinary stone disease is a highly prevalent condition, and the analysis of the size and volume of stones in the kidneys is an important point in surgical decisions and planning. ${ }^{16}$ Threedimensional stone segmentation software, out of non-enhanced computed tomography (CT) have been compared with radiologists readings and promise more accurate results. ${ }^{49}$ Also, approaches to identifying the composition of stones have been described, with the purpose of better metabolic management of urinary stones, and an accuracy of $100 \%$ was achieved in differentiating uric acid from non-uric acid stones in a study that compared different AI algorithms for CT scan interpretation. ${ }^{50}$

A review about the role of AI in urinary stone disease proposes these algorithms as the future of urology. Different methods are described, proposed to predict the probability of urinary stone disease out of symptoms, enhance the urinary stone tracking during shock wave lithotripsy (SWL) and minimize the number of emitted shockwaves during this procedure and also others that predict treatment success. ${ }^{16,51,52}$

Several ANNs have been designed to predict the outcomes after surgical interventions as SWL and percutaneous nephrolithotomy (PCNL). One of them used the information on 454 patients ( 200 for training set and 254 for test set), to assess the relevance of clinical preoperative parameters on postoperative results (PCNL) by comparing them to the actual (observed) outcomes; the accuracy and sensitivity of the system was found to range between $81 \%$ and $98.2 \%$, and it was able to predict stone-free rates with an accuracy of $86 \%{ }^{53}$ Stone-free rates after SWL were also evaluated by an ANN system based on information of 139 patients that was able to predict this outcome with an accuracy of $88.7 \% 54$

Finally, it is due to highlight the role of AI in surgical training. It has been studied mainly in robotic and laparoscopic surgery, with emphasis in anatomical landmark recognition as a fundamental step in automated surgery. ${ }^{55}$ Color and texture evaluation has been assessed during prostatectomy aiming to identify basic anatomical landmarks. ${ }^{56,57}$

Also, the identification of instruments and detection of its movement promises great results regarding prediction of surgical skill and technique. Khurshid et al reviewed the videos of 12 surgeons anastomoses (robotic radical prostatectomy) using the global evaluative assessment of robotic skills (GEARS) tool, initially manually, by 25 peer surgeons and compared the scores with the results of a linear support 
vector machine (SVM), which achieved an accuracy in classification of surgical skill of $83.3 \%$ and improved to $91.7 \%$ when joint movement was assessed. Finally, when the contralateral instrument was evaluated, accuracy was $100 \%{ }^{58}$

\section{Conclusions}

Urology is a constantly changing specialty. Each urologist has the responsibility to update him- or herself with the novel breakthroughs in the field, be aware of new pharmaceutical developments, and, in this century, they need not only to be aware, but be involved in the cutting-edge engineer and technological advances related to their specialty. All of the aforementioned pharmaceutical targets, genomic markers and technological innovations must be analyzed objectively, taking into account costs to the health systems, risks and benefits to the patients, and the legal background that comes with them. Technological advances, such as artificial intelligence and deep learning algorithms, have shown promising results but could not replace physicians' perspectives at the time. Certainly, our clinical practice needs to be retailored, and, with time, precision medicine is going to become the only possible medicine, tailoring treatments according to the genomic expression profile of each patient. Nowadays, research needs to be strengthened to make us reconsider changing the way we assess and treat our patients.

\section{Conflict of Interests}

The authors have no conflict of interests to declare.

\section{References}

1 Roychowdhury S, Chinnaiyan AM. Advancing precision medicine for prostate cancer through genomics. J Clin Oncol 2013;31(15): 1866-1873

2 Considine B, Petrylak DP. Novel Targets and Precision Medicine for Prostate Cancer-Part 2: Tumor Profiling and Personalized Therapy in Patients With Castration-Resistant Prostate Cancer. Oncology (Williston Park) 2019;33(04):128-131

3 Zumsteg ZS, Spratt DE. Precision Medicine for Localized Prostate Cancer: Time to Move Beyond NCCN Risk Stratification? Int J Radiat Oncol Biol Phys 2019;103(01):92-94. Doi: 10.1016/j. ijrobp.2018.09.040 [Internet]

4 Nuhn P, De Bono JS, Fizazi K, et al. Update on Systemic Prostate Cancer Therapies: Management of Metastatic Castration-resistant Prostate Cancer in the Era of Precision Oncology. Eur Urol 2019;75 (01):88-99. Doi: 10.1016/j.eururo.2018.03.028 [Internet]

5 Abbod MF, Catto JWF, Linkens DA, Hamdy FC. Application of artificial intelligence to the management of urological cancer. J Urol 2007;178(4 Pt 1):1150-1156

6 Narayan VM, Dahm P. The future of clinical trials in urological oncology. Nat Rev Urol 2019;16(12):722-733. Doi: 10.1038/ s41585-019-0243-x [Internet]

7 Harmon SA, Tuncer S, Sanford T, Choyke PL, Türkbey B. Artificial intelligence at the intersection of pathology and radiology in prostate cancer. Diagn Interv Radiol 2019;25(03):183-188

8 Venkatesan M. Artificial Intelligence vs. Machine Learning vs. Deep Learning. Data Sci Cent 2018;2Available from: https://www. datasciencecentral.com/profiles/blogs/artificial-intelligence-vsmachine-learning-vs-deep-learning

9 Ghani KR, Zheng K, Wei JT, Friedman CP. Harnessing big data for health care and research: are urologists ready? Eur Urol 2014;66 (06):975-977. Doi: 10.1016/j.eururo.2014.07.032 [Internet]
10 Bulten W, Pinckaers H, van Boven $\mathrm{H}$, et al. Automated deeplearning system for Gleason grading of prostate cancer using biopsies: a diagnostic study. Lancet Oncol 2020;21(02):233-241. Doi: 10.1016/S1470-2045(19)30739-9 [Internet]

11 Shkolyar E, Jia X, Chang TC, et al. Augmented Bladder Tumor Detection Using Deep Learning. Eur Urol 2019;76(06):714-718. Doi: 10.1016/j.eururo.2019.08.032 [Internet]

12 Nagpal K, Foote D, Liu Y, et al. Development and validation of a deep learning algorithm for improving Gleason scoring of prostate cancer. NPJ Digit Med 2019;2(01 [Internet] . Doi: 10.1038/ s41746-019-0112-2

13 Ali AM, Zhuang H, Ibrahim A, Rehman O, Huang M, Wu A. A machine learning approach for the classification of kidney cancer subtypes using miRNA genome data. Appl Sci (Basel) 2018;8(12):

14 Tabibu S, Vinod PK, Jawahar CV. Pan-Renal Cell Carcinoma classification and survival prediction from histopathology images using deep learning. Sci Rep 2019;9(01):10509. Doi: 10.1038/ s41598-019-46718-3 [Internet]

15 Poirion OB, Chaudhary K, Garmire LX. Deep Learning data integration for better risk stratification models of bladder cancer. AMIA Jt Summits Transl Sci Proc 2018;2017:197-206

16 Jahrreiss V, Veser J, Seitz C, Özsoy M. Artificial intelligence: the future of urinary stone management? Curr Opin Urol 2020;30 (02):196-199

17 Athie A, Arce-Gallego S, Gonzalez M, et al. Targeting DNA Repair Defects for Precision Medicine in Prostate Cancer. Curr Oncol Rep 2019;21(05):42

18 Nadal R, Bellmunt J. Management of metastatic bladder cancer. Cancer Treat Rev 2019;76(76):10-21. Doi: 10.1016/j. ctrv.2019.04.002 [Internet]

19 Akanksha M, Sandhya S. Role of FGFR3 in urothelial carcinoma. Iran J Pathol 2019;14(02):148-155

20 Loriot Y, Necchi A, Park SH, et al; BLC2001 Study Group. Erdafitinib in locally advanced or metastatic urothelial carcinoma. $\mathrm{N}$ Engl J Med 2019;381(04):338-348

21 Cancer P, Psma-based G, Psma L. Preparing Your Practice for the New Era of Theranostics. 2015;4(03):7-10

22 Motzer RJ, Tannir NM, McDermott DF, et al; CheckMate 214 Investigators. Nivolumab plus Ipilimumab versus Sunitinib in advanced renal-cell carcinoma. N Engl J Med 2018;378(14): $1277-1290$

23 Marcus L, Lemery SJ, Keegan P, Pazdur R. FDA approval summary: Pembrolizumab for the treatment of microsatellite instabilityhigh solid tumors. Clin Cancer Res 2019;25(13):3753-3758

24 Motzer RJ, Escudier B, McDermott DF, et al; CheckMate 025 Investigators. Nivolumab versus everolimus in advanced renalcell carcinoma. N Engl J Med 2015;373(19):1803-1813

25 Sharma P, Siefker-Radtke A, de Braud F, et al. Nivolumab alone and with ipilimumab in previously treated metastatic urothelial carcinoma: CheckMate 032 nivolumab $1 \mathrm{mg} / \mathrm{kg}$ plus ipilimumab $3 \mathrm{mg} / \mathrm{kg}$ expansion cohort results. J Clin Oncol 2019;37(19):1608-1616

26 Sharma P, Retz M, Siefker-Radtke A, et al. Nivolumab in metastatic urothelial carcinoma after platinum therapy (CheckMate 275): a multicentre, single-arm, phase 2 trial. Lancet Oncol 2017;18(03): 312-322

27 Motzer RJ, Penkov K, Haanen J, et al. Avelumab plus axitinib versus sunitinib for advanced renal-cell carcinoma. N Engl J Med 2019; 380(12):1103-1115

28 Powles T, Durán I, van der Heijden MS, et al. Atezolizumab versus chemotherapy in patients with platinum-treated locally advanced or metastatic urothelial carcinoma (IMvigor211): a multicentre, open-label, phase 3 randomised controlled trial. Lancet 2018;391(10122):748-757

29 Rini BI, Powles T, Atkins MB, et al; IMmotion151 Study Group. Atezolizumab plus bevacizumab versus sunitinib in patients with previously untreated metastatic renal cell carcinoma (IMmotion151): a multicentre, open-label, phase 3, randomised controlled trial. Lancet 2019;393(10189):2404-2415 
30 Rini BI, Plimack ER, Stus V, et al; KEYNOTE-426 Investigators. Pembrolizumab plus axitinib versus sunitinib for advanced renalcell carcinoma. N Engl J Med 2019;380(12):1116-1127

31 Bellmunt J, de Wit R, Vaughn DJ, et al; KEYNOTE-045 Investigators. Pembrolizumab as second-line therapy for advanced urothelial carcinoma. N Engl J Med 2017;376(11):1015-1026

32 Balar AV, Castellano D, O'Donnell PH, et al. First-line pembrolizumab in cisplatin-ineligible patients with locally advanced and unresectable or metastatic urothelial cancer (KEYNOTE-052): a multicentre, single-arm, phase 2 study. Lancet Oncol 2017;18(11):1483-1492. Doi: 10.1016/S1470-2045(17)30616-2 [Internet]

33 Stenehjem DD, Hahn AW, Gill DM, et al. Predictive genomic markers of response to VEGF targeted therapy in metastatic renal cell carcinoma. PLoS One 2019;14(01):e0210415

34 Mateo J, Porta N, Bianchini D, et al. Olaparib in patients with metastatic castration-resistant prostate cancer with DNA repair gene aberrations (TOPARP-B): a multicentre, open-label, randomised, phase 2 trial. Lancet Oncol 2020;21(01):162-174. Doi: 10.1016/S1470-2045(19)30684-9 [Internet]

35 Sandhu SK, Hussain M, Mateo J, et al. PROfound: Phase III study of olaparib versus enzalutamide or abiraterone for metastatic castration-resistant prostate cancer (MCRPC) with homologous recombination repair (HRR) gene alterations. Ann Oncol 2019;30 (November):ix188-ix189

36 Abida W, Campbell D, Patnaik A, et al. Preliminary results from the TRITON2 study of rucaparib in patients (pts) with DNA damage repair (DDR)-deficient metastatic castration-resistant prostate cancer (mCRPC): Updated analyses. Ann Oncol 2019;30(October):v327-v328. Doi: 10.1093/annonc/mdz248.003 [Internet]

37 Virtanen V, Paunu K, Ahlskog JK, Varnai R, Sipeky C, Sundvall M. PARP inhibitors in prostate cancer-The preclinical rationale and current clinical development. Genes (Basel) 2019;10(08):1-19

38 de Bono J, Mateo J, Fizazi K, et al. Olaparib for Metastatic CastrationResistant Prostate Cancer. N Engl J Med 2020;382(22):2091-2102

39 Abida W, Bryce AH, Vogelzang NJ, et al. 793PDPreliminary results from TRITON2: A phase II study of rucaparib in patients (pts) with metastatic castration-resistant prostate cancer (MCRPC) associated with homologous recombination repair (HRR) gene alterations. Ann Oncol 2018;29(Suppl 8):2018

40 Tan TZ, Rouanne M, Tan KT, Huang RYJ, Thiery JP. Molecular Subtypes of Urothelial Bladder Cancer: Results from a Metacohort Analysis of 2411 Tumors. Eur Urol 2019;75(03): 423-432. Doi: 10.1016/j.eururo.2018.08.027 [Internet]

41 Choi W, Porten S, Kim S, Willis D, Plimack ER, Roth B, et al. chemotherapy. 2015;25(02):152-165

42 Sartor AO, Morris MJ, Krause BJ. VISION: An international, prospective, open-label, multicenter, randomized phase 3 study of 177Lu-PSMA-617 in the treatment of patients with progressive PSMA-positive metastatic castration-resistant prostate cancer (mCRPC). J Clin Oncol 2019;37(15_suppl):TPS5099-TPS5099 [Internet] 10.1200/JCO.2019.37.15_suppl.TPS5099

43 Gaur S, Lay N, Harmon SA, et al. Can computer-aided diagnosis assist in the identification of prostate cancer on prostate MRI? a multi-center, multi-reader investigation. Oncotarget 2018;9(73): 33804-33817

44 Yang X, Liu C, Wang Z, et al. Co-trained convolutional neural networks for automated detection of prostate cancer in multiparametric MRI. Med Image Anal 2017;42:212-227

45 McGarry SD, Hurrell SL, Iczkowski KA, et al. Radio-pathomic Maps of Epithelium and Lumen Density Predict the Location of HighGrade Prostate Cancer. Int J Radiat Oncol Biol Phys 2018;101(05): 1179-1187

46 Chatterjee A, Bourne RM, Wang S, et al. Diagnosis of Prostate Cancer with Noninvasive Estimation of Prostate Tissue Composition by Using Hybrid Multidimensional MR Imaging: A Feasibility Study. Radiology 2018;287(03):864-873

47 Gorelick L, Veksler O, Gaed M, et al. Prostate histopathology: learning tissue component histograms for cancer detection and classification. IEEE Trans Med Imaging 2013;32(10):1804-1818

48 Nguyen K, Sarkar A, Jain AK. Prostate cancer grading: use of graph cut and spatial arrangement of nuclei. IEEE Trans Med Imaging 2014;33(12):2254-2270

49 Heiner S, Lieske J, Marcus R, Knoedler J, Dirks S, Fletcher J, et al. Use of dedicated stone analysis software to\&nbsp;assess urinary stone size: towards semi-automated metrics to enhance prediction of spontaneous stone passage. 2017

50 Kriegshauser JS, Paden RG, He M, et al. Rapid kV-switching singlesource dual-energy CT ex vivo renal calculi characterization using a multiparametric approach: refining parameters on an expanded dataset. Abdom Radiol (NY) 2018;43(06):1439-1445

51 Malmir B, Amini M, Chang SI. A medical decision support system for disease diagnosis under uncertainty. Expert Syst Appl 2017; 88:95-108

52 Shoar K, Turney BW, Cleveland RO. Tracking kidney stones in a homogeneous medium using a trilateration approach. J Acoust Soc Am 2017;142(06):3715-3721

53 Aminsharifi A, Irani D, Pooyesh S, et al. Artificial Neural Network System to Predict the Postoperative Outcome of Percutaneous Nephrolithotomy. J Endourol 2017;31(05):461-467

54 Seckiner I, Seckiner S, Sen H, Bayrak O, Dogan K, Erturhan S. A neural network- based algorithm for predicting stone - free status after ESWL therapy. Int Braz J Urol 2017;43(06):1110-1114

55 Chen J, Remulla D, Nguyen JH, et al. Current status of artificial intelligence applications in urology and their potential to influence clinical practice. Vol. 124, BJU International. Blackwell Publishing Ltd; 2019:567-577

56 Nosrati MS, Amir-Khalili A, Peyrat JM, et al. Endoscopic scene labelling and augmentation using intraoperative pulsatile motion and colour appearance cues with preoperative anatomical priors. Int J CARS 2016;11(08):1409-1418

$57 \mathrm{Al} \mathrm{O}$, Alawamlh H, Flannigan R, et al. Modeling automated assessment of surgical performance utilizing computer vision: proof of concept. J Urol 2018;199(42):e1134

58 Ghani KR, Liu Y, Law H, Miller DC, Montie J, Deng J. Video analysis of skill and technique (vast): machine learning to assess surgeons performing robotic prostatectomy. J Urol 2017;197(4S):e891 